\title{
Investigation of pressure retarded osmosis power production
}

\author{
Nikolaos Taousanidis ${ }^{1, *}$, and Konstantinos Gavros ${ }^{2}$ \\ ${ }^{1}$ Mechanical Engineering \& Industrial Design Dpt, Western Macedonia University of Applied \\ Sciences, Koila Kozani, Kozani, 50100, Greece \\ ${ }^{2}$ Electrical Engineering Dpt, Western Macedonia University of Applied Sciences, Koila Kozani, \\ Kozani, 50100, Greece
}

\begin{abstract}
A major source of energy exists where there is mixing between aqueous solutions of different salinities. This energy source is particularly concentrated where fresh water rivers flow on to the ocean. The power, represented by the osmotic pressure difference between fresh water and salt water, may be called salinity gradient power. In this study the pressure retarded osmosis method for the extraction of salinity gradients' energy is investigated, main problems and difficulties are pointed out and finally the whole subject is justified with experimental results.
\end{abstract}

\section{Introduction}

After decades of anticipation we may now be close to the ability to extract substantial amounts of energy from the sea. Serious efforts are underway in several countries to use the "free" energy of tidal oscillations, surface waves and mainly that resulting from thermal gradients. Even though a small portion of the solar radiative flux is directly deposited in the sea, the total energy budget of the ocean is large compared to man's needs. Some of the incident solar energy establishes and maintains thermal gradients. Water is evaporated, condensed and precipitated nonuniformly and establishes salinity differences.

This energy source exists at the interface between waters of differing salinities and is particularly concentrated where fresh water rivers flow on to the ocean. The power, represented by the osmotic pressure difference between fresh water and salt water, may be called salinity gradient power. Salinity power has certain features that make it attractive as a source of energy. It is large and untapped. Its employment could have little environmental impact. Compared with other sources of ocean energy, its density is high. It is renewable due to water evaporation by the sun and subsequent precipitation.

Experiments indicate that the equivalent pressure head between 0.5 molar seawater and fresh water is about $24 \mathrm{~atm}$. This pressure is equivalent to a $240 \mathrm{~m}$ water head. One may conceptualize it is a $240 \mathrm{~m}$ waterfall at the mouth of every river. Various techniques for the extraction of this form of energy has been proposed or developed. In this study we deal with the pressure retarded osmosis (PRO) technique [1].

${ }^{\star}$ Corresponding author: taousan@teiwm.gr 


\section{Principle of pressure retarded osmosis}

Osmosis is spontaneous transport of solvent molecules from a dilute solution to a more concentrated solution across a semipermeable membrane. In 1976, Sidney Loeb proposed pressure retarded osmosis (PRO) as a novel application for osmotic membranes [1]. The oil prices at that time were low and therefore the interest in further development of this technology was low. However, in 1997 Thorsen and Holt started a project together with the Norwegian company Statkraft [2, 3] and the world's first PRO pilot plant started in 2009.

PRO utilizes the osmotic pressure difference between two sources of different salinity to perform work and thus produce energy. The relationship between Reverse Osmosis (RO), Pressure Retarded Osmosis (PRO) and Direct Osmosis (DO) is illustrated in Figure 3.1 for an ideal semipermeable membrane. In DO, the membrane separates two solutions of unequal concentration, typically a salt solution from pure water. A flow of water from the water side into the salt solution takes place due to the difference in osmotic pressures between the two solutions. If an increasing pressure is applied to the salt solution, the magnitude of the water flow will decrease until no flow occurs when the applied pressure equals the osmotic pressure difference across the membrane. The regime in which there is a flow of water into a pressurized salt solution is known as pressure retarded osmosis. If the water volumetric permeation rate through the membrane, $\Delta \dot{V}$, is divided by membrane area, we have the water permeation flux, $J_{w}$. The relation between water permeation flux and applied forces, in the ideal case, is given by [8]:

$$
J_{W}=A(\Delta \pi-\Delta P),
$$

where $\mathrm{A}$ is the membrane water permeation coefficient, $\Delta \pi$ the osmotic pressure difference across the membrane and $\Delta \mathrm{P}$ is the hydrostatic pressure difference across the membrane.

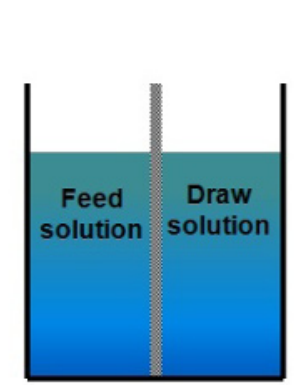

A) Equilibrium

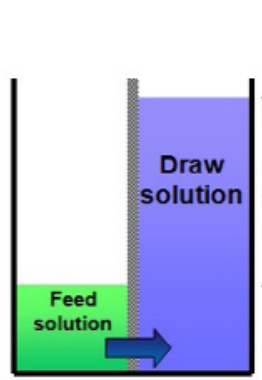

B) $F O(\Delta P=0)$

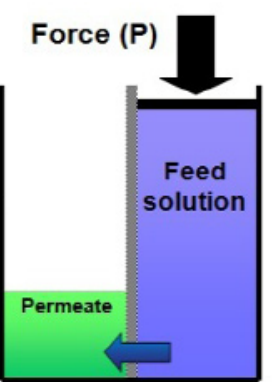

C) $R O(\Delta \mathrm{P}>\Delta \pi)$

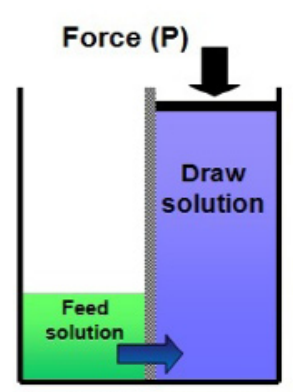

D) $\operatorname{PRO}(\Delta \mathrm{P}<\Delta \pi)$

Fig. 1. Classifications of osmotic pressures: a. osmotic equilibrium, b. DO $(\Delta P=0)$, c. RO $(\Delta P>\Delta \pi)$ and d. PRO $(\Delta P<\Delta \pi)[5]$

It is clear from equation (1) that if $\Delta \mathrm{P}=0$ we have direct osmosis (DO), for which $\mathrm{J}_{\mathrm{w}}$ $=\mathrm{A} \Delta \pi$. However if $0<\Delta \mathrm{P}<\Delta \pi$ we have Pressure Retarded Osmosis (PRO), so defined because the direction of permeation flux is still the same as in direct osmosis but the flux is decreased as the hydraulic pressure increases (in contrast to reverse osmosis (RO), a well developed technology, for which $\Delta \mathrm{P}>\Delta \pi$ and in which an increase in $\Delta \mathrm{P}$ increases the flux). PRO water permeates against the hydraulic pressure gradient, i.e., the flux is "uphill". The subsequent depressurization of the permeate through a hydroturbine-generator set would produce power by what may be described as an "osmotic waterfall".

The maximum power per unit flux is obtained at the maximum hydrostatic pressure under which PRO takes place, i.e., the osmotic pressure difference $\Delta \pi$. However, when $\Delta \mathrm{P}$ is close to $\Delta \pi$ the transmembrane water flux is very small, and a very large membrane area 
would be required, resulting in a high capital cost, being preferable, to operate PRO systems under maximum power per unit membrane area conditions.

The power per unit membrane area, $\mathrm{W}$ that can be generated in PRO is the product of the water flux across the membrane and the hydrostatic pressure of the salt solution [8]:

$$
W=J_{w} \Delta P=A(\Delta \pi-\Delta P) \Delta P
$$

By differentiating equation (2) with respect to $\Delta \mathrm{P}$, it can be shown that $\mathrm{W}$ reaches a maximum when $\Delta \mathrm{P}=\Delta \pi / 2$. Substituting this value for $\Delta \mathrm{P}$ in equation (2) yields

$$
W_{\max }=A \Delta \pi^{2} / 4
$$

According equation (3) the maximum power in a PRO system is directly proportional to water permeability coefficient $\mathrm{A}$, and thus high flux membranes are preferred. It is also proportional to the square of the osmotic pressure difference. This arises because increasing the osmotic pressure of the salt solution increases both the optimum pressure at which the system operates (i.e. $\Delta \pi / 2$ ) and the water flux through the membrane at that pressure.

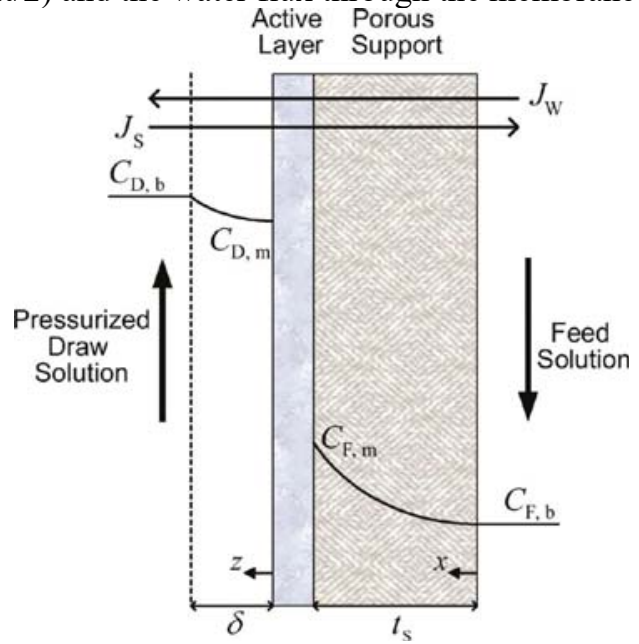

Fig. 2 Concentration profile over the membrane, direction $J_{\mathrm{w}}$ of the water flux and the salt flux Js are also shown [7]

The case is particularly different in real membranes as a series of reducing effects exist. They include salt leakage due to salt permeation in the 'opposite' direction and concentration polarization. While the first one is not considered a major problem, although reduces the effective osmotic pressure difference, the latter one is more severe. Concentration polarization occurs externally on the two sides of the membrane due to accumulation or depletion of solutes (external polarization) and internally in the necessary bulky porous substrate (internal polarization). Disturbing the boundaries on the two sides of the membrane we can minimizes external polarization. However this does not affect internal concentration polarization. Figure 2 schematically represents the results of these reducing effects upon concentrations and consequently upon osmotic pressures.

Equation (1) can then be modified to

$$
J_{w}=A\left(\Delta \pi_{e f f}-\Delta P\right)=A\left(\pi_{D, m}-\pi_{F, m}-\Delta P\right) .
$$

Equation (4) is not a useful expression for the water flux across a PRO membrane because the osmotic pressure is not known. As Lee et al. [8], outline the water flow can be accessed by the equation. 


$$
J_{W} \approx A\left(\frac{\pi_{2}}{1+B K}-\Delta P\right)
$$

where $\mathrm{B}$ is the salt permeation coefficient and $\mathrm{K}$ is a measure of the resistance to salt transport in the porous substrate. In this model, used as a basis also by other researchers [6], [7], membranes parameters $\mathrm{A}$ and $\mathrm{B}$ could be determined from $\mathrm{RO}$ while $\mathrm{K}$ can be obtained from $\mathrm{DO}$ measurements where $\Delta \mathrm{P}=0$ and pure water is used on one side of the membrane, i.e. $\mathrm{C}_{\mathrm{F}, \mathrm{m}}=0$. In this study the above model will be used and proved experimentally for novel design membranes, in the direction to overcome the commercial viable limit of $5 \mathrm{~W} / \mathrm{m}^{2}$ [4]. To date, no commercial membrane has demonstrated a power density larger than $3.5 \mathrm{~W} / \mathrm{m}^{2}$.

\section{Experiment}

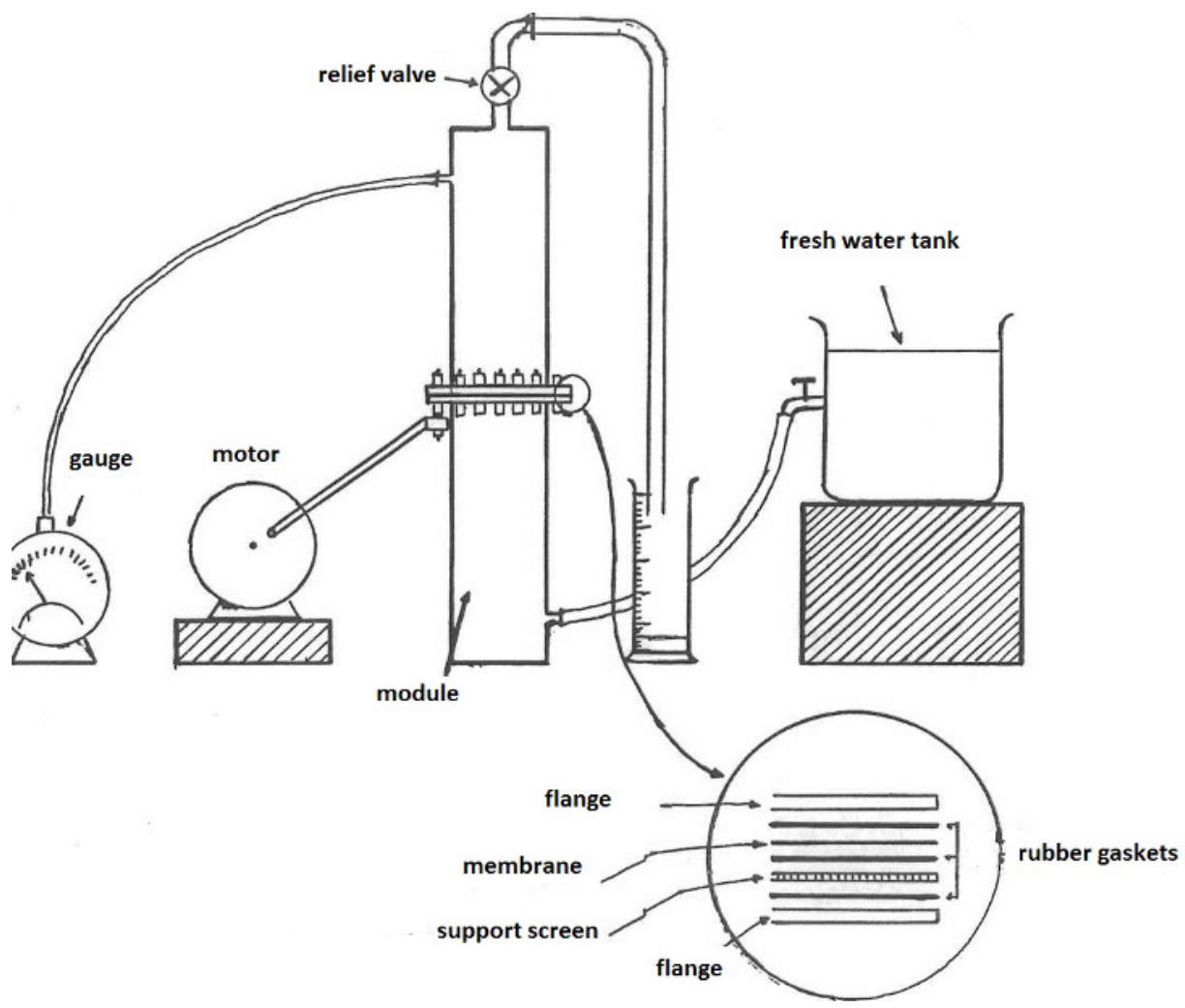

Fig. 3. Schematic drawing of the apparatus and circuit used for the experiment.

The basic apparatus used for the experiment was a cylindrical stainless steel pressure vessel. It contains the membrane, a support stainless steel screen for the membrane and three rubber gaskets, as shown in Figure 3, to make the whole assembly leak - proof. All of them (membranes, screen and gaskets) are between the two flanges, each one of each chamber, the whole module consisting of the upper and lower chamber. The upper chamber is the salt water chamber and the lower chamber is the fresh water chamber. The salt water chamber is provided with a pressure gauge. Also the outlet of the diluted solution from this chamber is provided with a relief needle valve. The outlet we have just named, lead the diluted solution to a graduated glass tube. Thus, we could measure the permeate volume and with the aid of a simple chronometer to calculate the permeate rate $\Delta \dot{V}(\mathrm{ml} / \mathrm{min})$ of 
fresh water through the membrane into the salt water chamber. The fresh water chamber is connected via a plastic tube to a fresh water tank, which continuously supplies water to the module to achieve a continuous operation.

Considering the external polarization, we put glass marbles in the upper chamber and plastic balls in the lower. The former because of their weight and the latter because of the water buoyancy come both very close to the membrane. We connected the apparatus via an eccentric aim to a low speed electric motor. When the power is on, the vessel is shaken vigorously by the arm resulting to elimination of the boundary layers which assist to the concentration polarization, moving both the glass marbles and the plastic balls.

Two HTI-sheet Cellulose Triacetate membranes of a very thin asymmetric cellulose acetate salt rejecting skin and an open - structured polysulfone porous substrate, were investigated. Both have salt rejection index of $95,6 \%$ for a $0,5 \% \mathrm{NaCl}$ aqueous solution and operating conditions: $\mathrm{pH}$ range $1-11$, temperature range $0{ }^{\circ} \mathrm{C}-85^{\circ} \mathrm{C}$, operating pressure $0-80$ bar for the HTI-CTA-011 and 0-50 for HTI-CTA-013 and water capacity is $451 / \mathrm{m}^{2} \mathrm{~h}$ for the first and $701 / \mathrm{m}^{2} \mathrm{~h}$ for the second. As the latter proved much better we present the results just for the HTI-CTA-013.

The system was fresh water $-3.5 \%$ wt. Aqueous $\mathrm{NaCl}$ solution. The experiment was undertaken, after the membrane was freed from the conservative medium (glycerine) by emerging it in the water for about 60 minutes and the original backing was carefully removed. That, improves the short - term efficiency of the membrane (time ranges of minutes) but causes a quick deterioration of the rest of the membrane.

The membrane has been used for a period of not more than two hours every day for about one week. With these time constraints we tried to minimize salt leakage and concentration polarization. The disturbance caused by the stirring effect of the combination of the low speed motor and the plastic and glass balls, interferes the growth of any boundary layer of different concentration than that in the chamber. The membrane requires about 50 minutes to reach the steady state flux, because there is not salt leakage when we start the experiment and the salt - water solution has not yet been diluted.

The effects of concentration polarization on water flux are illustrated in Table 1.

Table 1. Fluxes for both cases of continuous and interrupted agitation and power outputs

\begin{tabular}{|c|c|c|c|c|c|c|c|}
\hline \multirow{2}{*}{$\begin{array}{c}\text { Time } \\
\text { (min) }\end{array}$} & \multicolumn{2}{|c|}{ INTERRUPTED AGITATION } & \multicolumn{4}{c|}{ CONTINUOUS AGITATION } \\
\cline { 2 - 8 } & $\begin{array}{c}\text { Readings } \\
\text { flow in ml) }\end{array}$ & $\begin{array}{c}\text { flow rate } \\
(\mathrm{ml} / \mathrm{min})\end{array}$ & $\begin{array}{c}\text { flux of water } \\
\left(\mathrm{ml} / \mathrm{m}^{2} \text { min) }\right.\end{array}$ & $\begin{array}{c}\text { Readings } \\
\text { (flow in ml) }\end{array}$ & $\begin{array}{c}\text { flow rate } \\
(\mathrm{ml} / \mathrm{min})\end{array}$ & $\begin{array}{c}\text { flux of water } \\
\left(\mathrm{ml} / \mathrm{m}^{2} \text { min) }\right.\end{array}$ & $\begin{array}{c}\text { Work } \\
\text { watts x } 10^{-6}\end{array}$ \\
\hline 0 & - & & & - & & & \\
\hline 10 & 10 & 1.00 & 0.02325 & 10 & 1.00 & 0.02325 & 2.49567 \\
\hline 20 & 30 & 2.00 & 0.0465 & 30 & 2.00 & 0.0465 & 9.98268 \\
\hline 20 & 58 & 2.80 & 0.0651 & 58 & 2.80 & 0.0651 & 19.56606 \\
\hline 40 & 92 & 3.40 & 0.07905 & 92 & 3.40 & 0.07905 & 28.84996 \\
\hline 50 & 130 & 3.80 & 0.08835 & 130 & 3.80 & 0.08835 & 36.0375 \\
\hline 60 & 170 & 4.00 & 0.093 & 170 & 4.00 & 0.093 & 39.99307 \\
\hline 70 & 202 & 3.20 & 0.0744 & 208 & 3.80 & 0.08835 & 36.0375 \\
\hline 80 & 227 & 2.50 & 0.0581 & 248 & 4.00 & 0.093 & 39.99307 \\
\hline 90 & 249 & 2.20 & 0.05115 & 288 & 4.00 & 0.093 & 39.99307 \\
\hline 100 & 272 & 2.30 & 0.05347 & 329 & 4.10 & 0.09533 & 41.95664 \\
\hline 110 & 300 & 2.80 & 0.0651 & 368 & 3.90 & 0.0906 & 57.8964 \\
\hline
\end{tabular}

When the stirring - shaking was stopped, the water flux decreased markedly. This is the result of a higher salt concentration at the interface between the salt solution and the membrane than in the bulk solution and the development of a diluted salt solution layer near the membrane at the fresh water side. Resumption of stirring led to a sharp rise of 
water flux, surpassing the steady state value. This surge was real and reflected the condition in which virtually the entire osmotic pressure gradient was instantaneously imposed across the salt rejecting layer before the salt concentration profile could again develop within the membrane. However, the stirring cannot prevent the decline of the flux after a certain time that is the concentration polarization cannot be avoided ultimately.

Results show that the maximum flux is $0.09533 \mathrm{ml} / \mathrm{cm}^{2} \mathrm{~min}$. For $\mathrm{NaCl}$ concentration of about $3.5 \%$, molality is around 0.6 and for $15^{\circ} \mathrm{C}$, the theoretical osmotic pressure is about 26.4 bar. Thus, the water permeation coefficient can be calculated as follows:

$$
\mathrm{A}_{013}=0,09533 / 26,4=0,00361 \mathrm{ml} / \mathrm{cm}^{2} \min \mathrm{bar} .
$$

Knowing the A values and the fluxes from Table 1 we could form last column with the estimated values of power output from the following equation:

$$
W=J_{w} \Delta P=J_{w} J_{w} / A=J_{w}{ }^{2} / A .
$$

Thus the maximum power output is 41,95664 pWatts. These values are not the representative of the expected output from a salinity gradient source but illustrate the great significance and potential of these renewable energy forms.

\section{Conclusions}

1. Membrane properties under PRO conditions can be calculated using a model taking into account concentration polarization. Required parameters - water permeation coefficient $A$, salt permeation coefficient $B$, and resistance to salt transport in the membrane substrate $\mathrm{K}$ - can be obtained from RO and DO experiments.

2. Concentration polarization is a major problem in PRO. External concentration polarization can be minimized by stirring to reduce the thickness of boundary layers.

3. Internal concentration polarization can only be reduced to an acceptable level by using membranes with an open substrate. Without due regard for internal concentration polarization, it is unsafe to project PRO performance from RO performance.

4. Because PRO operating pressures with seawater/fresh water salinity gradient can be lower than those typically used in RO, compaction of the membranes due to the hydrostatic pressure gradient can be smaller in PRO, and PRO fluxes in the absence, of concentration polarization can be higher than high pressure RO data would suggest.

5. There is a serious prospect in energy extraction from salinity gradients if membrane problems could be resolved, attaining a production of minimum $5 \mathrm{~W} / \mathrm{m}^{2}$.

\section{References}

1. S. Loeb, , J Membrane Sci 1, 49-63 (1976)

2. K. Gerstandt, K.-V. Peinemann, S.E. Skilhagen, T. Thorsen, T. Holt, Desalination 224, 64-70 (2008)

3. T. Thorsen, T. Holt, J Membrane Sci 335, pp 103-110 (2009)

4. S.E.Skilhagen, Desalin Water Treat 15, 271-278 (2010)

5. I. L. Alsvik and M. Hägg, Polymers 5, pp 303-327 (2013)

6. A.Achilli, T.Y.Cath, A.E.Childress, Journal of Membrane Science 343, 42-52 (2009)

7. N.Yip, A.Tiraferri, W.Phillip, J. Schiffman, L.Hoover, Y.Kim, and M.Elimelech, Environ. Sci. Technol. 45, 4360-4369 (2011)

8. K.L. Lee, R.W. Baker, H.K. Lonsdale, J Membrane Sci 8, 141-171 (1981) 